\title{
Segmentierung der Kunden des Wiener Bio-Supermarktes „denn's Biomarkt“ mithilfe einer Clusteranalyse
}

\section{Customer segmentation of Viennese organic supermarket “denn's Biomarkt” using a cluster analysis}

\author{
Pia Sajovitz ${ }^{1}$ Siegfried Pöchtrager ${ }^{1}$, Tobias Truffner $^{1}$, Verena Auberger ${ }^{1}$
}

\begin{abstract}
1 Institut für Marketing und Innovation, Department für Wirtschafts- und Sozialwissenschaften, Universität für Bodenkultur Wien, Feistmantelstraße 4, 1180 Wien, Österreich

* Korrespondierende Autorin: pia.sajovitz@boku.ac.at
\end{abstract}

Einreichung: 28. Juli 2017, überarbeitete Einreichung: 11. Jänner 2018, Annahme: 15. Jänner 2018

\section{Zusammenfassung}

Obwohl der österreichische Bio-Fachhandel im Vergleich zum konventionellen Lebensmitteleinzelhandel nur einen geringen Anteil am Gesamtumsatz mit Bio-Lebensmitteln hat, nimmt die Anzahl der Bio-Fachhandelsgeschäfte konstant zu. Ziel der Arbeit war daher, die Kunden von Wiener Bio-Supermärkten (eine der Formen des Bio-Fachhandels) zu analysieren und auf Basis dieser Analyse Zielgruppen abzuleiten. Unter Verwendung eines standardisierten Fragebogens wurden 211 Kunden des Bio-Supermarktes „denn's“ befragt. Im Rahmen der Befragung wurden soziodemografische, psychografische und kaufverhaltensbezogene Merkmale ermittelt. Die Zielgruppensegmentierung der Teilnehmer erfolgte mittels Clusteranalyse unter Verwendung zweier Clusterverfahren (SingleLinkage- und Ward-Verfahren). Die deskriptiven Ergebnisse dieser Erhebung zeigen, dass die Kunden des Wiener Bio-Supermarktes „denn's“ vorwiegend weiblich sind. Allgemein sind die Kunden mittleren Alters und weisen ein vergleichsweise hohes Bildungs- und Einkommensniveau auf. Für die Befragten ist der Gesundheitsaspekt beim Bio-Lebensmitteleinkauf am relevantesten, gefolgt vom Umwelt- und Tierschutz. In Hinblick auf die Ernährungsgewohnheiten der Kunden sind über 60 \% Vegetarier oder Veganer und rund $35 \%$ achten auf eine gluten- oder laktosefreie Ernährung. Zudem sind die wesentlichsten Einkaufsmotive das Sortiment sowie Produktinformationen am Regal und eine deutliche Preiskennzeichnung. Mittels Clusteranalyse konnten zuvor vier unterschiedliche Kundenzielgruppen identifiziert werden.

Schlagworte: Bio-Lebensmittel, Bio-Supermärkte, Clusteranalyse, Konsumentenforschung, Zielgruppensegmentierung

\section{Summary}

Although the Austrian organic specialist trade only constitutes a small share of overall organic food revenues, the number of organic specialist trade shops has been increasing constantly. The aim of this study is to analyze customers of Viennese organic supermarkets (a type of organic specialist trade) and to derive target groups based on this analysis. Using a standardized questionnaire, 211 customers of organic supermarket "denn's" were surveyed. Within the analysis of the survey, sociodemographic and psychographic attributes as well as attributes related to buying behavior were detected. The target group segmentation was done by cluster analysis using two cluster methods (single linkage and ward method). Descriptive survey results show that customers of Viennese organic supermarket "denn's" are mainly female. Generally, customers are middle-aged and show a comparatively high level of education and income. When buying organic food, personal health is the most important aspect, followed by environmental protection and animal welfare. Regarding eating habits, more than $60 \%$ of the surveyed customers are vegetarians or vegans and about $35 \%$ follow a gluten- or lactose-free diet. Moreover, the crucial purchase motives are product selection, product information and pricing. Through cluster analysis four different target groups of customers were identified.

Keywords: organic food, organic supermarkets, cluster analysis, consumer research, target group segmentation 


\section{Einleitung}

Die Umsätze am europäischen Bio-Markt entwickeln sich seit Jahren konstant positiv. Im Vergleichszeitraum 2014 bis 2015 wuchsen die Bio-Handelsumsätze von 26,4 Mrd. $€$ um $13 \%$ auf 29,8 Mrd. €. Seit dem Jahr 2006, in welchem sich dieser Wert noch auf 13,9 Mrd. € belief, haben sich die Bio-Handelsumsätze sogar mehr als verdoppelt. Innerhalb Europas bildet Deutschland den größten BioMarkt. Im Jahr 2015 wurden in der deutschen Bio-Branche Bio-Lebensmittel und -Getränke im Wert von 8,62 Mrd. $€$ umgesetzt (Willer et al., 2017). Das Volumen des österreichischen Bio-Marktes betrug 2016 rund 1,64 Mrd. € (vgl. Bio Austria, 2017). Hinsichtlich des Pro-Kopf-Konsums lag im Jahr 2015 die Schweiz (262€ pro Kopf und Jahr) vor Dänemark (190 €) und Schweden (177 €). Österreich liegt in dieser Statistik mit $127 €$ auf dem sechsten Platz, wobei hier hervorgehoben werden muss, dass sich dieser Wert auf das Jahr 2011 bezieht. Deutschland weist mit $106 €$ einen geringeren Wert auf (Willer et al., 2017).

\section{Problemstellung, Zielsetzung und Forschungsfragen}

Von den in der Einleitung genannten 1,64 Mrd. €, die am Bio-Markt umgesetzt werden, entfielen 1,23 Mrd. $€$ auf den Lebensmitteleinzelhandel, 310 Mio. $€$ auf den Direktvertrieb und Fachhandel und 100 Mio. $€$ auf die Gastronomie (vgl. Bio Austria, 2017). In Österreich wächst der filialisierte Bio-Fachhandel insbesondere durch die deutsche Bio-Supermarktkette Dennree. Mit den „denn's Biomärkten“ besitzt sie derzeit 27 Filialen in Österreich, darunter 16 in Wien (Dennree Naturkost GmbH, 2018). Für ein erfolgreiches Marketing ist es notwendig, die Konsumentenzielgruppen von Bio-Lebensmitteln zu identifizieren und zu beschreiben. Ein Instrument hierfür bildet die Zielgruppensegmentierung, welche als zentrale Methode des strategischen Marketings gilt (Hamm et al., 2011). Ziel dieser Studie ist es, eine Einteilung der Kunden in Bio-Supermärkten zu treffen, indem deren Beweggründe für den Einkauf in Bio-Supermärkten identifiziert werden. Zusätzlich werden aktuelle Konsumtrends und Trends aus der Bio-Branche in die Befragung integriert. Die Arbeit basiert auf folgenden Forschungsfragen:

Wodurch zeichnen sich die Kunden des Wiener Bio-Supermarktes "denn's" aus?

Welche Marketingmaßnahmen sind allgemein und basierend auf der Zielgruppensegmentierung der „denn's“-Kunden empfehlenswert?

\section{Theoretische Grundlagen}

\subsection{Konsumenten von biologischen Lebensmitteln}

Verschiedenste Studien haben bereits versucht, soziodemografische Kriterien der Konsumenten von Bio-Lebensmitteln zu identifizieren. So wurde beispielsweise anhand einer Literaturanalyse festgestellt, dass Frauen tendenziell eher zu Bio-Lebensmitteln greifen als Männer (Aertsens et al., 2009). Die höhere Bio-Kaufintensität bei Frauen wird auch von Cordts et al. (2010) bestätigt. Hughner et al. (2007) konnten in ihrer Literaturanalyse einen steigenden Bio-Konsum im Alter feststellen, wohingegen Buder et al. (2010) keinen signifikanten Einfluss des Alters auf die Kaufintensität ermittelt haben. Diese unterschiedlichen Ergebnisse sind auf der einen Seite auf das steigende Gesundheits- und Ernährungsbewusstsein im Alter zurückzuführen. Auf der anderen Seite zieht der sich relativ rasch entwickelnde Bio-Markt immer mehr junge Menschen an (BÖLN, 2013). Knapp 50 \% der Bio-Supermarktkunden leben in Ein- oder Zweipersonenhaushalten (Kennerknecht et al., 2007). Die Studienergebnisse sind sich einig, dass ein positiver Zusammenhang zwischen dem verfügbaren monatlichen Haushaltseinkommen und der Kaufintensität von Bio-Lebensmitteln besteht (Wier et al., 2008). Auch Cordts et al. (2010) konnten sowohl das Haushaltsnettoeinkommen als auch das Bildungsniveau als signifikant in Bezug auf den Bio-Konsum identifizieren.

Die betrachteten Literaturanalysen über den Bio-Konsum kommen zum Schluss, dass das Gesundheitsmotiv ein zentrales Kriterium für den Kauf von Bio-Lebensmitteln darstellt (Hughner et al., 2007; Aertsens et al., 2009; Hemmerling et al., 2015). In Bezug auf den österreichischen Markt stehen für die Umfrageteilnehmer der RollAMA Motivanalyse Gesundheit und gesunde Ernährung an erster Stelle beim Kauf von Bio-Lebensmitteln. Aspekte wie Vermeidung von chemischen Zusatzstoffen und der Verzicht auf Spritzmittel sind am zweitwichtigsten, während sich Regionalität an dritter Stelle befindet. Des Weiteren bewegt der in den Augen der Umfrageteilnehmer bessere Geschmack von Bio-Produkten diese zum Kauf. Außerdem wurden von den Befragten noch Umwelt- und Klimaschutz, die bessere Qualität der Produkte sowie eine artgerechte Tierhaltung als Kaufmotive angeführt (AMA, 2015). Der bessere Geschmack, der Umweltschutz sowie artgerechte Tierhaltung werden auch von anderen Studien als äußerst relevant identifiziert (Hughner et al., 2007; Aertsens et al., 2009; Hemmerling et al., 2015). 
Die Kunden in Bio-Supermärkten schätzen ein Verkaufspersonal, welches sie gegebenenfalls persönlich beraten kann. Das Personal in Bio-Supermärkten sollte daher kontinuierlich geschult werden, um den Beratungsanforderungen der Kunden gerecht zu werden. Freundlichkeit und Kompetenz des Personals sind für Kunden von BioSupermärkten ebenso von Relevanz. Zur Förderung dieser Attribute nennen Kennerknecht et al. (2007) nicht-monetäre und monetäre Anreizsysteme, die der Steigerung der Mitarbeitermotivation dienen sollen. Ein wichtiger Faktor für Konsumenten biologischer Lebensmittel ist ein breites und innovationsorientiertes Bio-Sortiment. Sortimentsbreite und -tiefe stellen somit einen wichtigen Aspekt für die Kundenbindung bei Bio-Supermärkten dar. In den vergangenen fünf Jahren zeigte sich zudem auch eine Steigerung des Trockensortimentanteils am Gesamtangebot, was auf ein steigendes Kundeninteresse hinweist und daraus resultierend auf eine Wandlung vom Schwerpunkt auf Frischeprodukte hin zu einem umfangreichen Gesamtangebot deutet. Bei den Frischeprodukten werden vor allem Bio-Milchprodukte, -Obst, -Gemüse und -Fleisch vermehrt gekauft (Schaack, 2016).

Spezielle Ernährungsformen spielen für Konsumenten von Bio-Lebensmitteln häufig eine entscheidende Rolle. Laut einer IFES-Studie aus dem Jahr 2013 folgen in Österreich $9 \%$ der Bevölkerung einer vegetarischen oder veganen Lebensform (Vegane Gesellschaft Österreich, 2014). Ein anderes Bild zeichnet die Österreichische Gesundheitsbefragung 2014. Hier gaben lediglich 2,6\% der österreichischen Bevölkerung an, vollständig auf den Konsum von Fleisch- und Wurstwaren zu verzichten. Außerdem konstatierten 6,1\%, keinen Fisch zu verzehren (Statistik Austria, 2015). Nach einer Leserumfrage der Bio-Zeitschrift "Schrot \& Korn“ bei 4280 Kunden des deutschen BioFachhandels ernähren sich $23 \%$ der Befragten vegetarisch und $8 \%$ vegan. Die Umfrageteilnehmer gaben das Angebot veganer Produkte auch als bedeutendes Einkaufsmotiv an (Becker, 2016).

Legen Konsumenten gegenüber einem bestimmten Gut ein hohes Involvement an den Tag, äußert sich dies primär durch verstärkte Informationssuche und hohe kognitive Anstrengungen. Intensivkäufer, also solche die ein- bis mehrmals pro Woche Bio-Lebensmittel erwerben, zeichnen sich häufig durch diese Informationsnachfrage aus. Spiller et al. (2005) kamen in ihrer Studie zu dem Ergebnis, dass sich rund $48 \%$ der Umfrageteilnehmer ein- bis mehrmals pro Woche und ca. $8 \%$ sogar täglich über $\mathrm{Na}$ turkost informieren. Laut Ergebnissen des Ökobarometers
2013 beziehen Bio-Konsumenten ihre Informationen vor allem über private Kontakte zu anderen Bio-Konsumenten (60\%), über Beratung und Werbung in Bioläden oder bei Erzeugern (41\%), über Hörfunk- und TV-Beiträge sowie Zeitungsartikel (je 39 \%). Ebenso sind Artikel in Fachzeitschriften zur Informationsbeschaffung von Bedeutung (29\%). Das Internet spielt mit $16 \%$ nur eine untergeordnete Rolle (BÖLN, 2013).

\subsection{Marketingmaßnahmen für Bio-Konsumenten}

Die bereits in Kapitel 2.1 erwähnte Sortimentsbreite und -tiefe stellen für Kunden von Bio-Supermärkten wichtige Kaufmotive dar (Kennerknecht et al., 2007). Auch das Angebot von Alternativprodukten für Kunden, die sich vegan ernähren oder unter einer Nahrungsmittelunverträglichkeit leiden, ist für die Sortimentsgestaltung essenziell. Sabersky (2016) nennt hier beispielsweise laktose- und glutenfreie Produkte, Spezialmehle, Spurenmineralstoffe oder fermentierte Lebensmittel.

Meistens sind es eigennützige Motive (gesundheitliche und geschmackliche Vorzüge), die Konsumenten zum Kauf von Bio-Lebensmitteln bewegen. Da diese jedoch objektiv schwer nachzuweisen sind, eignen sich für die Kommunikationspolitik emotional angelegte Kampagnen besser als rational/informative. Der Grund dafür liegt darin, dass die Argumente schnell angreifbar und unglaubwürdig werden, wenn eine höhere geschmackliche sowie gesundheitliche Qualität nicht belegt werden kann (Schöberle, 2012). Eine Möglichkeit der Verkaufsförderung im Bio-Handel stellen gezielte Themenwochen $\mathrm{zu}$ wichtigen Bio-Kaufmotiven wie beispielsweise artgerechte Tierhaltung, Umweltschutz oder Gesundheit dar (Spiller et al., 2010).

\section{Methode und Stichprobenbeschreibung}

Die Befragung der Kunden fand im Zeitraum zwischen 6.-17. Juni 2016 mithilfe eines Fragebogens in fünf verschiedenen „denn's-Biomarkt“-Filialen in Wien statt, die hinsichtlich ihrer Filialanzahl die bedeutendste Bio-Supermarktkette in Österreich ist, worin auch die Auswahl als Befragungsort begründet liegt. Dabei wurden die Teilnehmer im Kassabereich angesprochen und gebeten, den Fragebogen selbstständig auszufüllen. Insgesamt füllten 211 Kunden den Fragebogen aus. 69 \% der Befragten sind weiblich, der Rest männlich. Rund 30 \% gehören der Altersklasse der 18- bis 29-Jährigen an und je ca. $20 \%$ der 
30- bis 39- bzw. 50- bis 59-Jährigen. Das Durchschnittsalter aller Befragten beträgt 41,6 Jahre. Die meisten Personen leben in Zweipersonenhaushalten (40,8 \%) und Einpersonenhaushalten (22,7 \%). Einen Abschluss an einer Hochschule bzw. Universität besitzen 66,8 \% der Befragten. $21 \%$ haben einen Maturaabschluss und $7 \%$ absolvierten eine berufsbildende mittlere Schule. Die Angestellten bilden mit rund $40 \%$ die größte Berufsgruppe, gefolgt von den Studenten und selbständig Erwerbstätigen mit je rund $20 \%$. Beamte bzw. Vertragsbedienstete und Pensionisten machen zusammen rund $17 \%$ der befragten „denn's“-Kunden aus. Die Arbeiter bilden mit 1,4\% die kleinste Berufsgruppe. Der größte Anteil der Befragten (18\%) verfügt über ein monatliches Netto-Haushaltseinkommen von unter $1000 €$. An zweiter Stelle befindet sich die Gruppe mit einem Haushaltseinkommen zwischen 1500 und $1999 €(16,1 \%)$. Mit 14,2\% auf dem dritten Platz liegen Kunden mit einem monatlichen Haushaltseinkommen von über $4000 €$. Die Anteile der drei Einkommensklassen zwischen 2000 und $3999 €$ sind ähnlich hoch. Somit besitzen rund $39 \%$ der befragten "denn's“Kunden ein monatliches Netto-Haushaltseinkommen von über $2500 €$.

Basierend auf den demografischen Kriterien „Ausbildung, Berufsgruppe und monatliches Netto-Haushaltseinkommen" wurde die Zugehörigkeit zu den sozialen Schichten errechnet. Die Berechnung der sozialen Schicht erfolgte auf Grundlage eines von der Gesellschaft für Konsumforschung (GfK) entwickelten Punkteverfahrens, angepasst an österreichische Verhältnisse. Die Unterteilung umfasst fünf Kategorien und reicht von A bis E, wobei A mit 435-582 Punkten die höchste und E mit 68-150 Punkten die niedrigste Schicht charakterisiert. In diesem Punkteverfahren werden die höchste abgeschlossene Ausbildung, die Berufsgruppe sowie das monatliche Haushaltsnettoeinkommen berücksichtigt. Hinsichtlich der Ausbildung reicht die Punkteanzahl von 20 (Pflichtschule) bis 120 (Hochschule, Universität). Bei den Berufsgruppen erhalten Studenten und Lehrlinge die geringste (20) und selbständig Erwerbstätige (70) die höchste Punkteanzahl. Das Punktespektrum des monatlichen Haushaltsnettoeinkommens erstreckt sich von unter $1000 €$ (65 Punkte) bis über $4000 €$ (391 Punkte) (GfK, 2009). Die Vorteile dieser Methode sind nach Meinung der Autoren die leichte Anwendung und die gute Vergleichbarkeit der Werte. Als Nachteil muss hingegen erwähnt werden, dass die Zuordnung zu einer Schicht keine Aussagen über Interessen, Einstellungen und Motive treffen kann. Anhand dieser Berechnungen kann festgestellt werden, dass ein Drittel $(31,3 \%)$ der teilnehmenden „denn's“-Kunden der A-Schicht angehört. An zweiter Stelle befinden sich die Kunden aus der B-Schicht mit 28,4\%, gefolgt von den Kunden aus der C-Schicht $(24,6 \%)$. Befragte aus der definierten D-Schicht bilden mit 15,6 \% die kleinste Gruppe. Der E-Schicht gehört indes keine Person der Stichprobe an. Die Auswertung der Schichtzugehörigkeit zeigt somit eine starke Konzentration der Oberschicht und oberen Mittelschicht unter den Teilnehmern.

Die Datenauswertung erfolgte mit dem Statistikprogramm IBM SPSS Statistics 21. In SPSS wurde mit den erhobenen Befragungsdaten eine Clusteranalyse für die Zielgruppensegmentierung der „denn's“-Kunden durchgeführt. Das Ziel der Clusteranalyse ist, Nachfrager basierend auf der Ähnlichkeit ihrer Merkmalsausprägungen in Gruppen zusammenzufassen. Die gebildeten Gruppen, sogenannte Cluster, sollten im Zusammenhang mit den betrachteten Eigenschaften oder Merkmalen möglichst homogen sein. Untereinander sollten die Gruppen jedoch eine hohe Heterogenität aufweisen, sich demnach möglichst voneinander unterscheiden. Die Zusammensetzung der Gruppen ist dem Anwender im Ausgangspunkt unbekannt, daher zählt die Clusteranalyse zu den explorativen Verfahren der multivariaten Analysemethoden. Ebenfalls relevant bei der Clusterbildung ist, dass alle betrachteten Merkmalsausprägungen gleichzeitig in die Gruppenbildung miteinbezogen werden (Freter, 2008; Meffert et al., 2015; Backhaus et al., 2016). Zur Clusterbildung wurden das Single-Linkageund das Ward-Verfahren angewendet. Um zu ermitteln, ob sich die Mittelwerte der Cluster signifikant unterscheiden, wurde eine einfaktorielle Varianzanalyse in Verbindung mit einem Post-hoc-Test durchgeführt. Als Post-hoc-Test wurde der Scheffé-Test angewendet. Dieser Test gehört zu den gängigsten Post-hoc-Tests und ist auch gültig, wenn die Stichprobenumfänge zwischen den Clustern wie im vorliegenden Beispiel unterschiedlich groß sind (Backhaus et al., 2016).

\section{Ergebnisse}

\subsection{Psychografische Merkmale}

Essenziell für die Bildung der Cluster sind die psychografischen Merkmale der befragten Konsumenten, da sich Personen einer demografisch ähnlichen Gruppe häufig in ihren Ansichten und Einstellungen unterscheiden (Kotler 
et al., 2003). Zu psychografischen Merkmalen zählen unter anderem Einstellungen, Motive und Interessen.

Anhand einer geschlossenen Frage wurden die Kunden gebeten, die Werte "Gesundheit“, „Geschmack“ und „Umwelt-/Tierschutz" nach ihrer Priorität zu reihen. Für rund $60 \%$ ist „Gesundheit" der relevanteste Aspekt beim Kauf von biologischen Lebensmitteln, für ca. 30 \% „Umwelt-/ Tierschutz" und für $10 \%$ der „Geschmack“. Letzterer besitzt im Umkehrschluss für $64 \%$ die geringste Bedeutung, gefolgt von „Umwelt-/Tierschutz“ (26\%) und „Gesundheit" $(10 \%)$.

Die befragten Kunden informieren sich mit unterschiedlicher Häufigkeit über Bio-Lebensmittel. So informieren sich $10 \%$ täglich und ca. $19 \%$ einmal bis mehrmals pro Woche. Rund $43 \%$ suchen einmal bis mehrmals im Monat nach Informationen über Bio-Lebensmittel. Seltener als einmal im Monat informieren sich $28 \%$ der Befragten. Unterschiedlichste Motive bewegen die befragten Konsumenten zum Einkauf im Bio-Supermarkt, wobei das Sortiment das bedeutendste Einkaufsmotiv darstellt. Rund 71 \% wählten die Antwortmöglichkeit „sehr wichtig“ und $27 \%$ „wichtig“. Auch die Kategorien „deutliche Kennzeichnung der Preise“ und „Produktinformationen am Regal“ stellen wichtige Einkaufsmotive dar. Beide Kategorien wurden von den befragten „denn's“-Kunden zu rund $82 \%$ mit den Antwortmöglichkeiten „sehr wichtig“ oder „wichtig“ bewertet. Die Möglichkeit, spezielle Produkte (z. B. vegane/ vegetarische Produkte, allergenfreie Produkte, verschiedene Öle, spezielle Getreidesorten/Brot und Gebäck, Kosmetika, frisches Obst und Gemüse, Fairtrade-Produkte, regionale/ saisonale Produkte, sämtliche Milchprodukte) im Bio-Supermarkt erwerben zu können, bildet für $45 \%$ ein sehr wichtiges Einkaufsmotiv (für 28,9 \% wichtig) und auch die allgemeine Atmosphäre im Geschäft (27\% wählten die Antwort „sehr wichtig“ und $45 \%$,wichtig“) ist für die Kunden bedeutend. Eine kompetente Beratung im Bio-Supermarkt wird von $57,2 \%$ als zumindest „wichtig“ erachtet. „Weniger wichtig“ bis ,überhaupt nicht wichtig“ sind für die befragten Personen die beiden Kategorien andere Kunden treffen und Produktverkostungen.

Vegetarisch ernähren sich rund $43 \%$ der Befragten und vegan ca. $19 \%$. Die Rohkost-Ernährung wird von 10,4 \% (22 Nennungen) befolgt. Auf eine glutenfreie Ernährung achten $19 \%$ und laktosefreie Produkte werden von $16 \%$ konsumiert.

In Bezug auf die Bedeutung bestimmter Eigenschaften von Bio-Lebensmitteln für die Kunden wählten 63 \% die Ant- wortmöglichkeit „am wichtigsten“ in der Kategorie „Gesundheit“. Die regionale Herkunft der Bio-Lebensmittel wird am häufigsten als zweitwichtigste Eigenschaft beim Einkauf von Bio-Lebensmitteln bewertet. Dennoch wurde die Regionalität auch von $28 \%$ als am wichtigsten eingestuft. Des Weiteren erachten 61 \% der befragten „denn's“Kunden den Preis als am drittwichtigsten und 25\% als am zweitwichtigsten. Der Convenience-Aspekt bei Bio-Lebensmitteln ist für die befragten Kunden von untergeordneter Bedeutung: Er wird von $84 \%$ als am unwichtigsten empfunden, wohingegen er für ca. $2 \%$ essenziell ist.

\subsection{Kaufverhaltensbezogene Merkmale}

Die Befragten bedienen sich unterschiedlicher Quellen, um an Informationen über Bio-Lebensmittel zu gelangen. Das Internet und private Kontakte werden mit ungefähr $62 \%$ bzw. $58 \%$ am häufigsten als Informationsquellen genutzt, gefolgt von Bio-Zeitschriften mit rund $40 \%$ und Massenmedien mit ca. $35 \%$. Allgemein nutzen $45 \%$ die Informationsangebote von „denn's Biomarkt“ bzw. „Dennree". Bio-Zeitschriften, welche im Kassenbereich der BioSupermärkte aufliegen (wie z. B. „Schrot \& Korn“, „eve“ etc.), besitzen für die befragten Kunden mit $29 \%$ die größte Relevanz. Am zweithäufigsten wird von $16 \%$ die Internetseite von Dennree als Informationsquelle genannt, gefolgt vom Newsletter (13\%). Die Facebook-Seite und der Bio-Blog von Dennree werden nur sehr selten von den befragten Kunden besucht bzw. gelesen.

Bezogen auf die Kaufhäufigkeit unterschiedlicher Produktgruppen im Bio-Supermarkt werden frisches Obst und Gemüse am häufigsten gekauft. Sowohl Obst als auch Gemüse werden zu rund $27 \%$ (Obst 27,1 \% und Gemüse $27,4 \%)$ mehrmals pro Woche eingekauft. Am zweithäufigsten werden Milchprodukte nachgefragt. Rund $12 \%$ der Befragten wählten die Antwortmöglichkeit „mehrmals in der Woche“ und 29,5 \% „einmal wöchentlich“. Eine weitere bedeutende Produktkategorie ist Brot und Gebäck. Diese wird von $11,5 \%$ mehrmals pro Woche und von $20,7 \%$ einmal in der Woche gekauft. Getreideprodukte und Teigwaren erwerben 21,6 \% (4,8 \% wählten „mehrmals pro Woche“ und 16,8 \% „einmal wöchentlich“) der befragten „denn's“-Kunden zumindest einmal wöchentlich. Die Kategorie „alkoholfreie Getränke“, dazu gehören auch Obst- und Gemüsesäfte, liegt bei der Einkaufshäufigkeit noch vor der Produktgruppe „Fleisch- und Wurstwaren“. Fertigprodukte und alkoholische Getränke, wie 
Wein und Bier, werden am seltensten von den befragten Kunden eingekauft. Ebenso wurde eruiert, wie viel Prozent ihrer monatlichen Lebensmittelausgaben die Befragten in Bio-Supermärkten ausgeben. Die Antwortmöglichkeit „10 bis $19 \%$ " wurde mit ca. $24 \%$ am häufigsten angekreuzt, gefolgt von der Kategorie „30 bis $49 \%$ “ mit rund $21 \%$. 20,6 \% der Befragten geben über die Hälfte ihrer Ausgaben für Lebensmittel in Bio-Supermärkten aus.

In Bezug auf die Einkaufshäufigkeit der Befragungsteilnehmer geben $38,4 \%$ an, einmal pro Woche und 28,4\% mehrmals in der Woche im Bio-Supermarkt einzukaufen. Ein- bis dreimal pro Monat kaufen 29,9 \% der Kunden ein und seltener als einmal pro Monat lediglich 3,3\%. In weiterer Folge werden die Kundengruppen in Intensivkäufer $(29,4 \%)$, Gelegenheitskäufer (39,7 \%) und Seltenkäufer (30,9\%) eingeteilt. Als Intensivkäufer gelten jene Personen, welche die Antwortmöglichkeit „mehrmals pro Woche" bei der Einkaufshäufigkeit in Bio-Supermärkten ankreuzten. Gelegenheitskäufer sind durch Kunden definiert, die einmal wöchentlich in Bio-Supermärkten einkaufen und Seltenkäufer ein- bis dreimal pro Monat. Somit entfallen für die weitere Clusteranalyse jene Umfrageteilnehmer, welche die Antwortmöglichkeit „seltener als einmal pro Monat" ausgewählt haben.

\subsection{Ergebnisse der Clusteranalyse}

Aufgrund ihrer Relevanz für die Zielgruppensegmentierung von Bio-Supermarktkunden und der guten Interpretierbarkeit wurden die Variablen des psychografischen Kriteriums „Einkaufsmotive“ analysiert. Nach dem Ausschluss von zwei Variablen, die nur eine geringe clusterbildende Wirkung besitzen, und dem Aussortieren von einem Ausreißer, blieben 210 Fallnummern zur Clusteranalyse übrig. Mithilfe des Single-Linkage- und des Ward-Verfahrens konnte die Clusteranzahl durch das Elbow-Kriterium auf vier Cluster festgelegt werden.

Abbildung 1 zeigt die Mittelwerte der einzelnen clusterbildenden Variablen, bezogen auf die jeweiligen Cluster. Die Werte eins bis vier auf der X-Achse stehen im Zusammenhang mit den Indikatorausprägungen der clusterbildenden Variablen ( 1 = sehr wichtig bis 4 = überhaupt nicht wichtig).

\section{Clusterinterpretation}

Cluster 1 - Die passiven „denn's“-Kunden mit speziellen Vorlieben $(n=87)$

Bezogen auf die Anzahl der Kunden ist dieser Cluster der größte. Für die Kunden des ersten Clusters ist die Atmo- sphäre im Geschäft im Vergleich am unbedeutendsten. Die deutliche Kennzeichnung der Preise ist für diese Kunden gleich relevant wie für jene des zweiten Clusters. Des Weiteren werden Produktverkostungen von den Kunden durchschnittlich mit ,weniger wichtig“ bewertet (Mittelwert = 3,0). Spezielle Produkte im Bio-Supermarkt einkaufen zu können, stellt für die Personen dieser Gruppe das wichtigste Einkaufsmotiv dar. Hierbei unterscheidet sich diese Gruppe auch signifikant vom dritten und vierten Cluster. Hinsichtlich der Käufergruppen besteht dieser Cluster zu jeweils $40 \%$ aus Gelegenheits- und Seltenkäufern und zu $20 \%$ aus Intensivkäufern. Das Durchschnittsalter ist mit 38 Jahren $(\sigma=13)$ im Vergleich zu den anderen drei Gruppen am niedrigsten. Hierbei bilden die 29- bis 49-Jährigen die größte Altersgruppe mit $42 \%$, gefolgt von den unter 29-Jährigen mit $37 \%$.

\section{Cluster 2 - Die neugierigen "denn's“-Kunden mit speziellen} Vorlieben $(n=55)$

Die Geschäftsatmosphäre und die kompetente Beratung durch das Verkaufspersonal sind für die Kunden des zweiten Clusters vergleichsweise am bedeutendsten. In diesen beiden Merkmalen bestehen auch signifikante Unterschiede zu den anderen drei Gruppen. Zudem werden von den Kunden dieses Clusters Produktverkostungen und Produktinformationen am Regal im Vergleich ebenfalls am relevantesten bewertet. Wie auch für die Personen des ersten Clusters stellen spezielle Produkte im Bio-Supermarkt ein bedeutendes Einkaufsmotiv dar, und es lassen sich signifikante Unterschiede zur dritten und vierten Gruppe feststellen.

Bezogen auf die Käufergruppen besteht dieser Cluster im Gegensatz zum ersten Cluster, der sich zu je $40 \%$ aus Gelegenheits- und Seltenkäufern zusammensetzt, aus jeweils $41 \%$ Intensiv- und Gelegenheitskäufern und zu $18 \%$ aus Seltenkäufern. Der Frauenanteil in diesem Cluster ist mit $75 \%$ vergleichsweise hoch und die Kunden über 50 Jahre bilden die größte Altersgruppe (40\% der Personen des Clusters).

Cluster 3-Die uninteressierten "denn's“-Kunden $(n=29)$

Der dritte Cluster ist hinsichtlich der Kundenanzahl der kleinste. Die Geschäftsatmosphäre ist für diese Kunden am relevantesten. Abgesehen von der Geschäftsatmosphäre und den speziellen Produkten wurden die vier restlichen Einkaufsmotive bzw. clusterbildenden Variablen vergleichsweise am unwichtigsten von den Kunden bewertet. Hinsichtlich Beratung, deutliche Preiskennzeichnung und Produktinformationen am Regal konnten signifikan- 
te Unterschiede zu den anderen drei Clustern festgestellt werden.

Die Käufergruppen sind mit jeweils $35 \%$ Intensiv- und Gelegenheitskäufern und $30 \%$ Seltenkäufern annähernd gleichmäßig verteilt. Zudem ist die Geschlechterverteilung mit $55 \%$ Frauen und $45 \%$ Männern vergleichsweise am ausgewogensten. Beim Durchschnittsalter, das 43 Jahre $(\sigma=17)$ beträgt, liegen die Kunden dieser Gruppe zwischen dem zweiten (44 Jahre, $\sigma=15$ ) und vierten (45 Jahre, $\sigma=13$ ) Cluster.

\section{Cluster 4-Die interessierten "denn's"-Kunden ( $n=39)$}

Die Geschäftsatmosphäre und die kompetente Beratung bewerten die Kunden des vierten Clusters durchschnittlich mit wichtig (Mittelwert $=2,0$ bzw. 2,1). Sie liegen somit bezüglich der Bedeutung dieser Einkaufsmotive bzw. clusterbildenden Variablen zwischen der Bewertung der zwei- ten sowie der ersten und vierten Gruppe. Die deutliche Preiskennzeichnung wird von den Kunden dieses Clusters im Vergleich als am wichtigsten eingestuft. Zudem legen diese Kunden großen Wert auf Produktinformationen am Regal. Hinsichtlich der sehr geringen Bedeutung von speziellen Produkten unterscheidet sich dieser Cluster signifikant von den anderen drei Clustern.

Der Frauenanteil der Kunden dieses Clusters ist mit 77 \% vergleichsweise am höchsten. Bei den Käufergruppen überwiegen die Gelegenheitskäufer mit $42 \%$, gefolgt von den Seltenkäufern (31\%) und Intensivkäufern (27\%).

\section{Diskussion und Schlussfolgerungen}

Die Diskussion der Ergebnisse erfolgt aufbauend auf den zwei in Kapitel 1 formulierten Forschungsfragen.

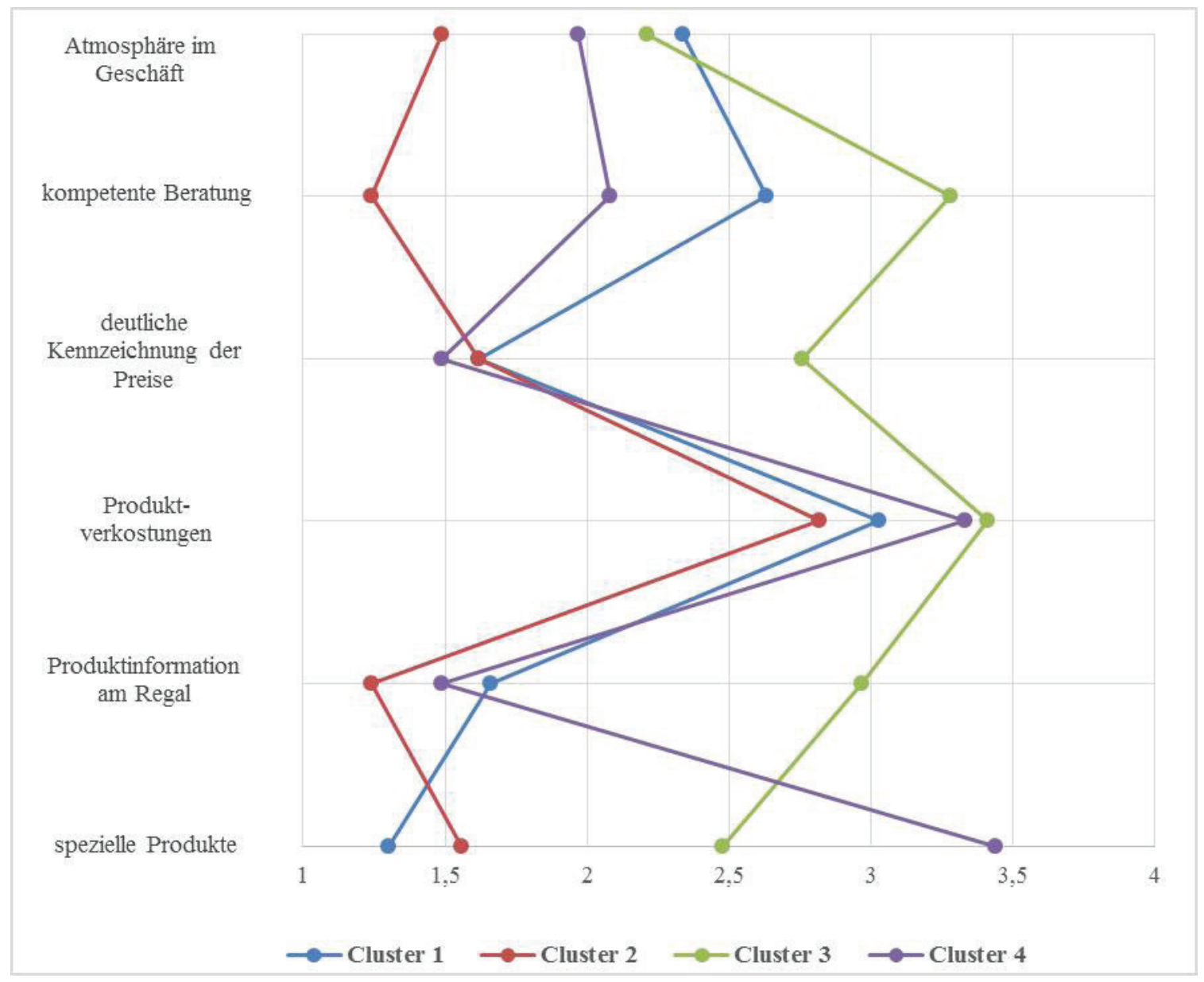

Abbildung 1. Imageprofil (Mittelwerte) der 4-Cluster-Lösung Figure 1. Image profile (mean values) of the 4-cluster solution 
1. Wodurch zeichnen sich die Kunden des Wiener Bio-Supermarktes "denn's" aus?

Im ersten Schritt der Beantwortung dieser Forschungsfrage sollen die soziodemografischen Merkmale der befragten „denn's“-Kunden diskutiert werden. Rund $69 \%$ aller Befragten in dieser Erhebung sind Frauen. Auch Aertsens et al. (2009) und Cordts et al. (2009) weisen auf eine allgemein höhere Bio-Kaufintensität bei Frauen hin. In Bezug auf das Alter bzw. die gebildeten Altersgruppen stellen die 18-29-Jährigen mit rund $30 \%$ die größte Gruppe dar, gefolgt von den 30-39- und 50-59-Jährigen mit jeweils rund $20 \%$. Die Befragung im Rahmen des Ökobarometer 2013 sieht bei deutschen Verbrauchern unter 30 Jahren eine wachsende Beliebtheit von Bio-Lebensmitteln (BÖLN, 2013). Des Weiteren leben die Umfrageteilnehmer zu rund $64 \%$ in Ein- oder Zweipersonenhaushalten. In der Bio-Supermarkt-Kundenbefragung von Kennerknecht et al. (2007) leben ebenfalls knapp 50 \% der Kunden in Einoder Zweipersonenhaushalten.

Hinsichtlich des Netto-Haushaltseinkommens verdienen rund $39 \%$ der Befragten mehr als $2500 €$ monatlich. Allgemein kann davon ausgegangen werden, dass ein positiver Zusammenhang zwischen dem verfügbaren Haushaltseinkommen und der Kaufintensität von Bio-Lebensmitteln besteht (Wier et al., 2008; Cordts et al., 2010). Widersprüchlich hierzu bilden die Haushaltseinkommen unter $1000 €$ die größte Einkommensklasse mit rund $18 \%$ der in dieser Studie befragten „denn's“-Kunden. Dies ist jedoch auf den hohen Studentenanteil von $20 \%$ in der Stichprobe zurückzuführen. Studenten machen rund $76 \%$ aller Befragten in der Einkommensklasse unter $1000 €$ aus. Viele Studenten gaben an, in Mehrpersonenhaushalten bzw. Wohngemeinschaften zu leben, daher erscheint ein NettoHaushaltseinkommen unter $1000 €$ als eher unrealistisch. Nach Meinung der Autoren gaben die befragten Studenten vermutlich ihr eigenes Einkommen anstatt des Haushaltseinkommens an. Obwohl die Netto-Haushaltseinkommen unter $1000 €$ die größte Einkommensklasse bildet, stammen bezogen auf die soziale Schichtzugehörigkeit insgesamt $60 \%$ der befragten Kunden aus der A-Schicht und aus der B-Schicht. Das Bildungsniveau ist allgemein sehr hoch, da rund $21 \%$ aller Befragten die Matura und $67 \%$ einen Universitätsabschluss besitzen. Sowohl das hohe Bildungsniveau als auch die höhere Schichtzugehörigkeit von Bio-Konsumenten wird auch von anderen Studien wie beispielsweise Kennerknecht et al. (2007), Wier et al. (2008) und Cordts et al. (2010) bestätigt.
In Bezug auf die Bedeutung verschiedener Werte beim BioLebensmitteleinkauf zeigt sich, dass der Gesundheitsaspekt für die meisten Befragten am wichtigsten ist. Der Tier- und Umweltschutz liegt bezüglich seiner Relevanz für die Teilnehmer am häufigsten an zweiter Stelle. Den teilweise als besser empfundenen Geschmack von Bio-Lebensmitteln stuft der Großteil der befragten Kunden als am drittwichtigsten ein. Auch in zahlreichen anderen Studien konnte der Gesundheitsaspekt für Bio-Konsumenten allgemein als am bedeutendsten beim Einkauf von biologischen Lebensmitteln identifiziert werden (Aertsens et al., 2009; AMA, 2015; Hemmerling et al., 2015). Der bessere Geschmack von Bio-Lebensmitteln liegt jedoch in verschiedenen Studien noch vor den Motiven Umwelt- und Tierschutz, wie die internationale Literaturanalyse von Hemmerling et al. (2015) zeigt.

Das Sortiment stellt für die befragten Kunden das wichtigste Motiv für den Einkauf im Bio-Supermarkt dar. Auch Kennerknecht et al. (2007) bestätigen, dass sich die Sortimentsbreite und -tiefe besonders positiv auf die $\mathrm{Zu}$ friedenheit der befragten Kunden auswirkt. Hinsichtlich der gekauften Produkte werden frisches Obst und Gemüse am häufigsten gekauft, gefolgt von Milchprodukten sowie Brot und Gebäck. Der Frischebereich im Bio-Fachhandel ist allgemein von großer Bedeutung für dessen Umsatz, auch wenn der Anteil des Trockensortiments zunimmt (Schaack, 2016).

In einer für die österreichische Bevölkerung repräsentativen Studie des Instituts für empirische Sozialforschung (IFES) aus dem Jahr 2013 geben $9 \%$ der Befragten an, sich vegetarisch oder vegan zu ernähren (Vegane Gesellschaft Österreich, 2014). In der vorliegenden Erhebung ist der Anteil an Vegetariern und Veganern hervorzuheben: Von den Teilnehmern verfolgen insgesamt $62 \%$ eine vegetarische oder vegane Ernährungsweise. Dieser Wert ist einerseits in Relation zur Gesamtbevölkerung Österreichs laut der Gesundheitsbefragung der Statistik Austria (2015) verzichten lediglich 2,6 \% zur Gänze auf Fleisch- und Wurstwaren - sehr hoch, andererseits auch verglichen mit den Ergebnissen anderer Studien. Eine Leserumfrage der Bio-Zeitschrift „Schrot \& Korn“ mit 4280 deutschen BioFachhandelskunden kam zum Ergebnis, dass sich $23 \%$ der Teilnehmer vegetarisch und $8 \%$ vegan ernähren. Zudem stellt das Angebot an veganen Produkten, wie auch in der vorliegenden Erhebung, ein bedeutendes Einkaufsmotiv für die befragten Bio-Fachhandelskunden dar (Becker, 2016). 
Hinsichtlich der Einkaufshäufigkeit im Bio-Supermarkt zählen rund $29 \%$ der Befragten zu den Bio-Intensivkäufern, ca. $40 \%$ zu den Bio-Gelegenheits- und $31 \%$ zu den Bio-Seltenkäufern. Auch die Verteilung, wie häufig sich die befragten Kunden über Bio-Lebensmittel informieren bzw. das daraus abgeleitete Involvement der Befragten, weist eine ähnliche Verteilung auf. Täglich bis einmal pro Woche informieren sich ca. 29 \% der Befragten und sind somit nach der Definition von Spiller et al. (2005) stärker involviert. Von den geringer involvierten Kunden, die im Zuge der vorliegenden Studie befragt wurden, beziehen rund $43 \%$ einmal bis mehrmals pro Monat Informationen über Bio-Lebensmittel und $28 \%$ seltener als einmal monatlich. Allgemein geben die Umfrageteilnehmer an, sich vorwiegend über das Internet (62\%), über ihr privates Umfeld (58\%) und verschiedene Bio-Zeitschriften (40\%) über biologische Lebensmittel zu informieren. Medien wie Fernsehen, Radio und Zeitungen werden am wenigsten genutzt. Bei den Ergebnissen des Ökobarometers 2013 hinsichtlich des Informationsverhaltens von deutschen Bio-Konsumenten sind private Kontakte ebenfalls sehr bedeutende Informationsquellen für biologische Lebensmittel. Auch Bio-Zeitschriften spielen eine wichtige Rolle, während in dieser Studie das Internet für die Befragten als Informationsquelle von geringer Bedeutung ist (BÖLN, 2013).

\section{Welche Marketingmaßnahmen sind allgemein und basie-} rend auf der Zielgruppensegmentierung der "denn's"-Kunden empfehlenswert?

Die eigene Gesundheit und die Gesundheit ihrer Mitmenschen stehen beim Bio-Lebensmitteleinkauf für die Befragten vorwiegend an erster Stelle. Bei der Kommunikationspolitik sollte daher speziell auf die Zusammenhänge zwischen gesunder Ernährung und dem Konsum von BioLebensmitteln eingegangen werden. Die gesundheitlichen Vorteile von biologischen Lebensmitteln sind jedoch oft nicht objektiv nachweisbar, daher ist in diesem Zusammenhang eher eine emotionale als eine rational/informative Kommunikationspolitik empfehlenswert (Schöberle, 2012). Altruistische Werte beim Bio-Lebensmitteleinkauf, wie der Umwelt- und Tierschutz oder die regionale Herkunft der Produkte, sind für die Befragten ebenfalls von großer Bedeutung. Diese sollten im Rahmen der Kommunikationspolitik ebenfalls verstärkt berücksichtigt werden. Das umfangreiche Sortiment des Bio-Supermarkts stellt für die Kunden das wesentlichste Einkaufsmotiv dar. Auch ausgewählte bzw. spezielle Produkte aus dem Sortiment sind für die Befragten von Relevanz. Bei der Sortimentsgestaltung empfiehlt es sich, die speziellen Ernährungsgewohnheiten der Kunden zu berücksichtigen. Den hohen prozentuellen Anteil der sich vegetarisch bzw. vegan ernährenden Kunden gilt es hierbei ebenso zu beachten wie den hohen Anteil an Konsumenten von gluten- und laktosefreien Produkten. Auch Alternativprodukte für Kunden mit Nahrungsmittelunverträglichkeiten und Veganer, wie beispielsweise Spezialmehle, fermentierte Lebensmittel oder Spurenmineralstoffe, sollten im Sortiment nicht fehlen (Sabersky, 2016). Zudem können insbesondere vegane Lebensmittel sowie gluten- und laktosefreie Produkte im Sortiment speziell und eindeutig gekennzeichnet und hervorgehoben werden, um den Kunden die Suche nach ihren gewünschten Produkten zu erleichtern. Auch die hohe Relevanz von regionalen und saisonalen Produkten für die Bio-Supermarktkunden konnte in dieser Erhebung festgestellt werden. Wo möglich, sollte der Anteil der regionalen Produkte im Sortiment erhöht werden. Des Weiteren empfiehlt sich eine spezielle Hervorhebung bei der Präsentation von regionalen Produkten im Bio-Supermarkt. In diesem Zusammenhang können auch Produktinformationen am Regal eingesetzt werden. Diese wurden von den Umfrageteilnehmern als zweitwichtigstes Einkaufsmotiv gewertet. Hierbei könnten beispielsweise Flyer zu regionalen oder saisonalen Produkten oder deren Herstellern am Regal angebracht werden.

Bezogen auf das Informationsverhalten der befragten „denn's“-Kunden hinsichtlich biologischer Lebensmittel stellen das Internet, private Kontakte und Bio-Zeitschriften die bedeutendsten Informationsquellen dar. Bio-Zeitschriften, wie beispielsweise das „bioMagazin“ oder „Schrot $\&$ Korn", werden auch am häufigsten aus dem Informationsangebot in den „denn's-Biomarkt“-Filialen genutzt. Für Bio-Supermarktbetreiber empfiehlt es sich daher, verstärkt in diesen Zeitschriften zu inserieren und die Vorteile des Einkaufens im Bio-Supermarkt gezielt zu bewerben. Ausreichend Zeitschriften sollten auch konstant im Kassenbereich zur freien Entnahme aufliegen. Aufgrund der großen Beliebtheit von Bio-Zeitschriften könnten von den BioSupermarktbetreibern auch monatlich Kundenzeitschriften veröffentlicht werden. Dieses Kundenmagazin könnte verschiedene Rezepte mit Produkten aus dem Sortiment sowie Informationen und Artikel zu Produkten und der Bio-Landwirtschaft allgemein enthalten.

Die Handlungs- bzw. Marketingempfehlungen für die identifizierten Zielgruppen sollen nachfolgend beispielhaft beschrieben werden. Für die Befragten des ersten Clusters 
sind bestimmte Produkte beim Einkauf im Bio-Supermarkt sehr relevant. Die angegebenen speziellen Produkte der Kunden dieses Clusters sind verschieden und reichen u. a. von bestimmten veganen und vegetarischen Produkten, über gluten- und laktosefreie Lebensmittel, bis hin zu Produkten, die in anderen Einkaufsstätten schwer zu finden sind. Zudem sind $80 \%$ der Kunden dieses Clusters Bio-Selten- oder Bio-Gelegenheitskäufer, deren Kaufhäufigkeit durch gezielte Marketingmaßnahmen erhöht werden könnte. Hierbei empfiehlt sich eine stetige Anpassung des Bio-Supermarktsortiments an die speziellen Vorlieben dieses Clusters. In der Kommunikationspolitik könnten sowohl das breite Sortiment als auch die Präsenz spezieller Produkte im Bio-Supermarkt durch verschiedene Kommunikationsinstrumente hervorgehoben werden. Auch Themenwochen zu unterschiedlichen Produkten bzw. Produktkategorien und Sonderaktionen hinsichtlich speziell nachgefragter Produkte könnten die Kunden dieses Clusters stärker ansprechen (Kennerknecht et al., 2007; Spiller et al., 2010; Sabersky, 2016).

Die Kunden des zweiten Clusters legen, neben der hohen Bedeutung von speziellen Produkten, Wert auf eine kompetente Beratung durch das geschulte Verkaufspersonal sowie Produktinformationen am Regal. Für sie ist ein kompetentes und freundliches Verkaufspersonal besonders relevant, welches gegebenenfalls diese interessierten Kunden beraten kann. Die Mitarbeiter sollten dahingehend möglichst gut geschult und ausgebildet werden. Kennerknecht et al. (2007) empfehlen, neben finanziellen auch nicht-monetäre Anreizsysteme zu entwickeln, um die Motivation des Verkaufspersonals in Bio-Supermärkten zu erhöhen. Dies könnte speziell für die Bio-Intensivkäufer dieses Clusters relevant sein. Beratung, Produktinformation und deutliche Preiskennzeichnung stellen auch für die Kunden des vierten Clusters wesentliche Einkaufsmotive dar und sollten daher nicht vernachlässigt werden.

Beim dritten Cluster handelt es sich um tendenziell uninteressierte „denn's“-Kunden. Die Geschäftsatmosphäre stellt jedoch im Vergleich zu den anderen Einkaufsmotiven ein bedeutendes Einkaufsmotiv dar. Eine angenehme Atmosphäre im Bio-Supermarkt kann daher besonders für diese Kunden relevant sein und zu einer Erhöhung der Einkaufshäufigkeit führen.

Zusammengefasst lässt sich auf Basis der Identifikation sowie Segmentierung der unterschiedlichen Zielgruppen die Relevanz angepasster Marketingmaßnahmen erkennen. Für die Entscheidungsträger des Bio-Fachhandels ist es wichtig, ihre Konsumentengruppen sowie über deren
Einstellungen, Kaufmotive und Interessen informiert zu sein, um durch gezieltes Marketing direkt und unmittelbar auf die sich wandelnden Kundenbedürfnisse reagieren zu können.

Anzumerken ist, dass sich die Studienergebnisse aufgrund der Stichprobengröße von 211 Personen auf den Befragungsort Wien beschränken und dementsprechend limitierend wirken.

\section{Literatur}

Aertsens, J., Verbeke, W., Mondelaers, K. und G. Van Huylenbroeck (2009): Personal determinants of organic food consumption: a review. British Food Journal 111, 1140-1167.

AMA - Agrarmarketing Austria (2015): RollAMA-Motivanalyse Biologische Lebensmittel. http://www.ama marketing.at/home/groups/4/Charts_Bio-Motivanalyse. pdf. Abgerufen am 3. Jänner 2018.

Backhaus, K., Erichson, B., Wulff, P. und R. Weiber (2016): Multivariate Analysemethoden - Eine anwendungsorientierte Einführung. 14., überarbeitete und aktualisierte Aufl., Springer Gabler, Berlin - Heidelberg.

Becker, N. (2016): Die Welt der Leser im Fokus. BioHandel 09, 47-49.

Bio Austria (2017): Statistik. http://www.bio-austria.at/ bio-bauern/statistik/. Abgerufen am 3. Jänner 2018.

BÖLN (2013): Ökobarometer 2013 - Repräsentative Bevölkerungsbefragung im Auftrag des Bundesministeriums für Ernährung, Landwirtschaft und Verbraucherschutz (BMELV). http://www.bmel.de/SharedDocs/ Downloads/Ernaehrung/Oekobarometer_2013.pdf. Abgerufen am 3. Jänner 2018.

Buder, F., Hamm U., Bickel, M., Bien, B. und P. Michels (2010): Dynamik des Kaufverhaltens im Bio-Sortiment. http://orgprints.org/16983/. Abgerufen am 3. Jänner 2018.

Cordts, A., Schulze, B., Padilla-Bravo, C.A. und A. Spiller (2010): Bio-Kaufverhalten und Bio-Marketing. In: Hoffmann, I. und A. Spiller (Hrsg.): Auswertung der Daten der Nationalen Verzehrsstudie II (NVS II): eine integrierte, verhaltens- und lebensstilbasierte Analyse des Bio-Konsums. http://orgprints.org/18055/. Abgerufen am 3. Jänner 2018.

Dennree Naturkost GmbH (2018): Filialsuche. http:// www.denns-biomarkt.at/710_ihr_denns_biomarkt. html. Abgerufen am 3. Jänner 2018. 
Freter, H. (2008): Markt- und Kundensegmentierung: Kundenorientierte Markterfassung und -bearbeitung. 2., vollständig neu bearbeitete und erweiterte Aufl., W. Kohlhammer Verlag, Stuttgart.

GfK - GfK Austria GmbH (2009): Studienergebnisse Weiterbildung in Österreich. http://www.sagedpw.at/ uploads/Studienergebnisse_WeiterbildunginOesterreich_552.pdf. Abgerufen am 7. Februar 2017.

Hamm, U., Hemmerling, S., Schleenbecker, R., Spiller, A. und S. Wägeli (2011): Wissensstandsanalyse zum Verbraucher- und Ernährungsverhalten bei ökologischen Lebensmittel.).:n mit Einbezug der Außer-Hausverpflegung. BÖLN, Frankfurt/M.

Hemmerling, S., Hamm, U. und A. Spiller (2015): Consumption behaviour regarding organic food from a marketing perspective - a literature review. Organic Agriculture 5, 277-313.

Hughner, R.S., McDonagh, P., Prothero, A., Shultz, C.J. und J. Stanton (2007): Who are organic food consumers? A compilation and review of why people purchase organic food. Journal of Consumer Behaviour 6, 94-110.

Kennerknecht, R., Spiller, A., Bolten, J. und A. Zühlsdorf (2007): Marketing im Naturkostfachhandel - Eine empirische Untersuchung zur Kundenzufriedenheit und Kundenbegeisterung. https://www.uni-goettingen.de/ de/...pdf/Marketing\%20im\%20Naturkostfachhandel. pdf. Abgerufen am 3. Jänner 2018.

Kotler, P., Armstrong, G., Saunders, J. und V. Wong (2003): Grundlagen des Marketing. 3. Aufl., Pearson Studium, München.

Meffert, H., Burmann, C. und M. Kirchgeorg (2015): Marketing - Grundlagen marktorientierter Unternehmensführung. 12., überarbeitete und aktualisierte Aufl., Springer Gabler, Wiesbaden.

Sabersky, A. (2016): Trends fürs Regal. BioHandel 09, 29-33.

Schaack, D. und H. Willer (2016): Organic Farming and Market Development in Europe. In: Willer, H. und J. Lernoud (Hrsg.): FiBL \& IFOAM - Organic International - The World of Organic Agriculture: Statistics and Emerging Trends 2016. IFOAM und FiBL, Bonn - Frick, 199-229.
Schaack, J. (2016): Situation im Naturkostfachhandel 2015. In: Moewius, J., Röhrig, P., Schaack, D., Brzukalla, H.-J., Sanders, J., Zandonella, R., Sutter, D., Stokar, v.T., Löwenstein, F. und R. Bickel (Hrsg.): Zahlen Daten Fakten - Die Bio-Branche 2016. BÖLW, Berlin, 12-13.

Schöberle, S. (2012): Verbraucherverhalten bei Bio-Lebensmitteln: Analyse des Zusammenhangs zwischen Einstellungen, Moralischen Normen, Verhaltensabsichten und tatsächlichen Kaufverhalten. Dissertation, Technische Universität München, München.

Spiller, A., Engelken, J. und S. Gerlach (2005): Zu Zukunft des Bio-Fachhandels: Eine Befragung von BioIntensivkäufern. Department für Agrarökonomie und Rurale Entwicklung, Universität Göttingen, http:// www.konsumwende.de/Dokumente/Biofachhandelsstudie.pdf. Abgerufen am 3. Jänner 2018.

Spiller, A., Wittig, F., Cordts, A., Claupein, E. und I. Hoffmann (2010): Fazit und Handlungsempfehlungen. In: Hoffmann, I. und A. Spiller (Hrsg.): Auswertung der Daten der Nationalen Verzehrsstudie II (NVS II): eine integrierte, verhaltens- und lebensstilbasierte Analyse des Bio-Konsums, S. 131-142. http://orgprints. org/18055/. Abgerufen am 3. Jänner 2018.

Statistik Austria (2015): Österreichische Gesundheitsbefragung 2014 - Hauptergebnisse des Austrian Health Interview Survey (ATHIS) und methodische Dokumentation. Selbstverlag, Wien.

Vegane Gesellschaft Österreich (2016): 9 \% leben vegetarisch oder vegan. https://vegan.at/inhalt/9-leben-vegetarisch-oder-vegan. Abgerufen am 3. Jänner 2018.

Wier, M., O’Doherty Jensen, K., Mørch Andersen, L. und K. Millock (2008): The character of demand in mature organic food markets: Great Britain and Denmark compared. Food Policy 33, 406-421.

Willer, H., Schaack, D. und J. Lernoud (2017): Organic Farming and Market Development in Europe and the European Union. In: Willer, H. und J. Lernoud (Hrsg.): The World of Organic Agriculture: Statistics and Emerging Trends 2017. Fibl \& IFOAM - Organics International, Frick - Bonn 207-243. 